\title{
Cross-correlations between CMEs and other Solar Activity Indices
}

\author{
W.B. Song $\dagger$ and J.X. Wang \\ National Astronomy Observatories, Chinese Academy of Sciences, Beijing 100012, China \\ email: wenbin@ourstar.bao.ac.cn
}

\begin{abstract}
Using the list of CMEs observed by SOHO/LASCO, we compile a daily CME counts from January 1996 to December 2003. Cross-correlations between the CME counts and other three solar activity indices, i.e., flare index, sunspot number, and photospheric magnetic flux, are examined in both real and Fourier spaces. We find that correlations are all significant in real space, but only photospheric magnetic flux has good correlation with CME counts in Fourier space. Typical periods of CME occurrence are presented and discussed.
\end{abstract}

Keywords. Sun: activity, coronal mass ejections (CMEs), flares, magnetic fields

\section{Data Analysis}

A study on CMEs is an important topic that relates directly to space environments. Here we take our emphasis on correlations between CME occurrence and other solar activity indices.

Figure $1 a$ has shown four daily sequences: CME counts $(C C)$, sunspot number $(S N)$, flare index $(F I)$ and photospheric magnetic flux $(P M F)$. The former three ones come from Websites, The last one is constructed by connecting NSO/Kitt Peak synoptic charts (Stenflo \& Güdel, 1988) and calculating magnetic flux like Ballester \& Oliver (2002). About five months long gaps of $C C$ are filled with a method introduced by Fahlman \& Ulrych (1982).

To assess the degree of correlation we compute cross-correlation coefficient $\omega$ at zero lag defined as $\omega=\frac{\sum_{j=1}^{M}\left(x_{j-1}-\bar{x}\right)\left(y_{j-1}-\bar{y}\right)}{\sqrt{\left[\sum_{j=1}^{M}\left(x_{j-1}-\bar{x}\right)^{2}\right]\left[\sum_{j=1}^{M}\left(y_{j-1}-\bar{y}\right)^{2}\right]}}$. To test for significance level we use F-test. Its corresponding function is $R_{c}=\sqrt{\frac{F_{c}(1, M-2)}{(M-2)+F_{c}(1, M-2)}}$, where $F_{c}(1, M-2)$ can be looked up in F-list at one definite level $(\alpha=0.99)$. If $\omega>R_{c}$, we can say that this correlation is significant, otherwise it can be considered due to chance.

\section{Results}

We examine the correlations both in real and Fourier spaces. In real space, from Table 1 we can find that three correlation coefficients are all positive and very significant. Besides, the coefficients between $C C$ and $S N, P M F$ are both rather high. In Fourier space, Figure $1 b$ has shown the normalized Fourier power spectra of these four sequences after zeromeaned, we compute the correlation coefficients in $0.005-0.05 /$ day frequency domain and get that $\omega(C C-F I)=0.0015, \omega(C C-S N)=-0.011, \omega(C C-P M F)=0.35$. Their corresponding $R_{c}$ are all about 0.23 . Therefore, only $P M F$ has good correlation with $C C$ in Fourier space. This is very different from what happened in real space. Moreover, from

$\dagger$ Present address: A20 Datun Rd., Chaoyang Dist., Beijing 100012, China 

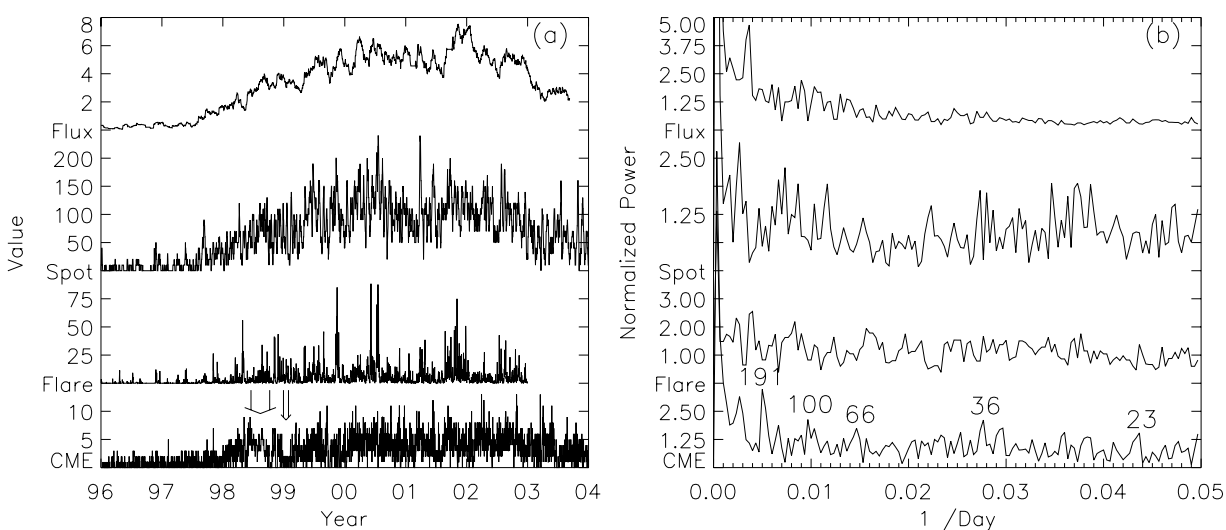

Figure 1. (a) Daily variations of $C C, F I, S N$ and $P M F$ from 1996 to 2003. Arrows point to the gaps of CMEs list. Here the $P M F$ unit is $10^{23} \mathrm{Mx}$; (b) Fourier power spectra of the four sequences shown in Figure $1 a$.

Figure $1 b$ we can find some typical periods in $C C$, including 191-day period, 100-day period, 66-day period, and so on. Many of them are also mentioned by Lou et al. (2003).

Table 1. Cross-correlations between $C C$ and $F I, S N, P M F$

\begin{tabular}{lccc}
\hline Solar Activity Indices & $M($ days $)$ & $\omega$ & $R_{c}$ \\
\hline Flare Index & 2557 & 0.28 & 0.067 \\
Sunspot Number & 2922 & 0.49 & 0.062 \\
Photospheric Magnetic Flux & 2812 & 0.55 & 0.063 \\
\hline
\end{tabular}

\section{Discussion}

We find that the correlations between $C C$ and $F I, S N, P M F$ are highly significant in real space. While in Fourier space we find that only the flux sequence has such good correlation. Therefore, we consider that pulses in $P M F$ can place a premium on CME occurrence. This may due to the fact that they both belong to global (or large scale at least) solar indices. It is really elusive that there is almost no correlation between $C C$ and FI in Fourier space. First, the CME and flare may have very different physical mechanisms, for example, the flare basically an active region phenomenon. Second, different definitions and measurements of such two indices cannot be ignored. That the fewer correlation in real space and non-correlation in Fourier space between $C C$ and $S N$ would be expected because their difference is obvious except solar cyclic changes.

\section{Acknowledgements}

This work is supported by National Natural Science Foundation of China (10233050) and National Key Basic Research Foundation of China (G2000078404).

\section{References}

Ballester, J. L., Oliver, R. \& Carbonell, M. 2002, ApJ 566, 505

Fahlman, G. G. \& Ulrych, T. J. 1982, MNRAS 199, 53

Lou, Y. Q., Wang, Y. M., Fan, Z. H., Wang, S. \& Wang, J. X. 2003, MNRAS 345, 809

Stenflo, J. O. \& Güdel, M. 1988, A\&\&A 191, 137 